\title{
DEVELOPMENT AND IN VIVO TESTING OF RECONFIGURABLE NEURAL PROBES FOR CHRONIC ELECTRICAL RECORDING Aalap Dighe $^{1}$, Ulrich P. Froriep ${ }^{1}$, Michael Sunshine ${ }^{2}$, Aiva Ievins ${ }^{2}$, Polina Anikeeval ${ }^{1}$, Chet Moritz ${ }^{2}$, and Joel Voldman ${ }^{1 *}$ \\ ${ }^{1}$ Massachusetts Institute of Technology, Cambridge, Massachusetts, USA \\ ${ }^{2}$ University of Washington, Seattle, Washington, USA
}

\begin{abstract}
We present the successful in vivo testing of a triggerable, reconfigurable neural probe designed to record neural activity in the central nervous system for extended periods of time. The probe consists of a thin polyimide-gold-polyimide sandwich structure that can be deflected prior to insertion to store mechanical energy. After inserting into the brain and waiting for the initial glial sheath to form, the device can be triggered, causing the recording tip of the device to penetrate into fresh tissue. Here we present the first in vivo triggering and acute electrical recordings from this device in the rodent motor cortex, demonstrating the essential device functionality.
\end{abstract}

\section{INTRODUCTION}

Electrical recording interfaces with the nervous system are critical for enabling neurally-controlled prostheses and better therapies for neurological disorders [1]. A major challenge with reliable chronic neural recording is tissue reaction, wherein a sheath of glial cells encapsulates the neural probe, electrically insulating the probe from the surrounding neurons [2]. This glial sheath arises both as a reaction to the initial surgical trauma and as a long-term reaction to the foreign body.

Various strategies have been tried to mitigate the glial reaction, including designing electrodes that are flexible [3], deliver immunosuppressants [3], or have bioactive coatings [4]. To-date, however, these approaches have not eliminated the chronic tissue response. Because of data suggesting that repositioning electrodes after the initial glial response has subsided can recover signals [5], and that small feature sizes reduce tissue response [6-8], researchers have started developing small reconfigurable probes, either using a Sandia surfacemicromachined process [9] or a triggerable variant of the Michigan probes [10]. (a)

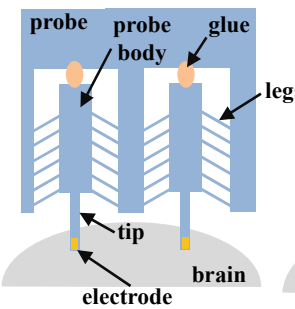

(b)

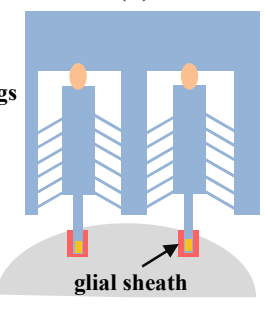

(c)

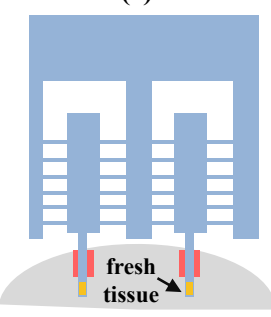

Figure 1: Device operation schematic. (a) The probe bodies are deflected and glued prior to insertion into the brain. (b) A glial sheath forms over time ( weeks) due to initial surgical trauma. (c) Dissolving the glue triggers the probe body to deflect the tips carrying recording electrodes into fresh tissue. Small tip dimensions prevent subsequent glial sheath formation.

Here we describe the first in vivo electrical recordings from a triggered reconfigurable probe with small dimensions. Our approach is to create a thin polymer probe whose body can be deflected and locked prior to insertion via a "glue", storing mechanical energy in the legs (Figure 1a). After inserting into the brain and waiting for the initial glial sheath to form (Figure 1b), the device can be triggered by dissolving the glue (Figure 1c), causing the recording tip of the device to penetrate into fresh tissue. Designing the tip dimensions to be small [8] $(7-20 \mu \mathrm{m})$ should mitigate formation of an additional glial sheath posttriggering and thus improve chronic recording.

\section{MODELING}

Analytical models of the structural mechanics of the device body were developed to inform optimal device materials and dimensions, in order to balance device flexibility (to reduce elastic modulus mismatch with the brain), stiffness (to allow piercing through the glial sheath without buckling), energy storage in the device legs (to provide the $>1 \mathrm{mN}$ force needed to pierce the glial sheath, based on similar triggered probes [9]) and adequate travel distance once deployed [11] ( $>50 \mu \mathrm{m}$, to move the recording sites past the thickness of the glial sheath). Figure 2 shows example model results informing the appropriate lengths of the device legs, given the tradeoff between the mechanical stress induced in the deflected device legs and the total force that can be delivered by the device.

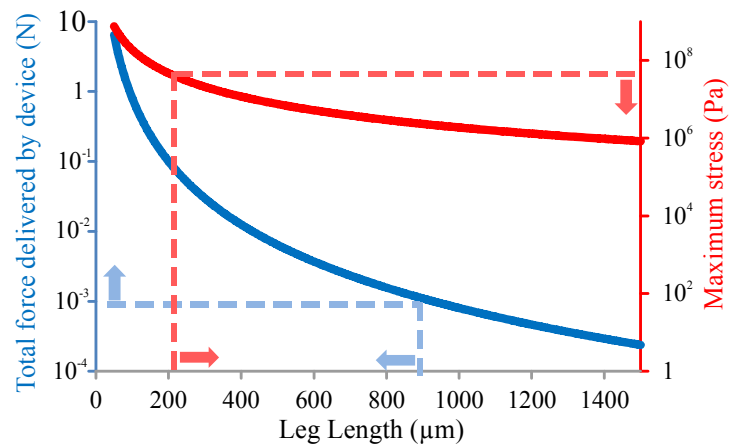

Figure 2: Plot of analytically calculated force delivered by the device (left) and the maximum stress in the device legs (right) as a function of leg length for a leg width of $20 \mu \mathrm{m}$, a leg thickness of $20 \mu \mathrm{m}$ and a deflection distance of $50 \mu \mathrm{m}$. The requirement that the force delivered be $\geq 1 \mathrm{mN}$ (design criteria) and the maximum stress be $\leq 70 \mathrm{MPa}$ (yield stress of polyimide) informs a design space for the leg length (between 200 and $900 \mu \mathrm{m}$ ).

Mechanical buckling theory was used to ensure a tip geometry that should be able to pierce through the brain tissue and the glial sheath without buckling. Electrical models were also used to determine appropriate electrode materials, electrode site sizes, and trace widths. Additionally, the chosen device materials and dimensions needed to be biocompatible and compatible with available microfabrication facilities. All mechanical and electrical models were validated with laser-cut polyimide film prototypes as 
well as microfabricated short loop tests (data not shown) before microfabrication of the final devices.

Based on the above models and prototypes, the devices were chosen to have a $10 \mu \mathrm{m}$ polyimide / $200 \mathrm{~nm}$ gold / $10 \mu \mathrm{m}$ polyimide sandwich structure. The structural material was chosen to be polyimide because of its biocompatibility [12], its flexibility (reducing mechanical modulus mismatch with the brain and allowing for increased travel distance, compared to silicon probes) and its ability to store adequate energy when deflected despite being flexible. The device leg widths were varied between $20-40$ $\mu \mathrm{m}$, the leg lengths were varied between $200-500 \mu \mathrm{m}$, and leg spacing was varied between $20-40 \mu \mathrm{m}$. The device tips were designed to be $20 \mu \mathrm{m}$ wide at the smallest point. The electrode site areas were varied between $450-5000 \mu \mathrm{m}^{2}$.

\section{FABRICATION}

The probe was fabricated using standard surface micromachining techniques, as shown in Figure 3.

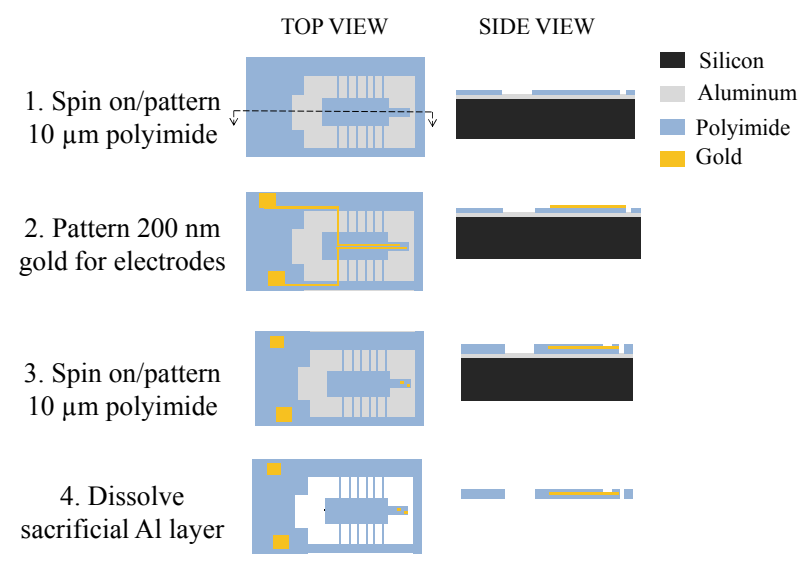

Figure 3: Fabrication process flow.

First, a $1-\mu \mathrm{m}$-thick sacrificial aluminum layer was sputterdeposited onto a 6-inch silicon wafer. Next, a 10- $\mu$ m-thick base layer of photo-patternable polyimide (HD 4100) was spun onto the wafer and patterned using photolithographic masks (step 1). The polyimide was then partially cured $\left(320^{\circ} \mathrm{C}\right.$ for $\left.30 \mathrm{~min}\right)$ to create a stable surface for subsequent processing, while leaving some unterminated bonds for attaching to the top polyimide layer. Next, a 10 -nm-thick titanium +200 -nm-thick gold layer was deposited using electron beam evaporation, and patterned using an acetone liftoff process (step 2). This gold layer forms the electrode sites and traces for the probe. The $10-\mu \mathrm{m}$-thick top layer of polyimide (HD 4100) was then spun onto the wafer and patterned. The wafer was hard baked $\left(360^{\circ} \mathrm{C}\right.$ for $\left.60 \mathrm{~min}\right)$ to complete the full cure of both polyimide layers (step 3). Finally, the polyimide sheet was removed from the silicon wafer by dissolving the sacrificial aluminum in aluminum etchant (Transene - type A) (step 4). All die geometries were designed so that the final dies could be easily separated by hand after peeling the polyimide film from the silicon wafer, thereby avoiding a final diesaw step. The fabricated device is shown in Figure 4.

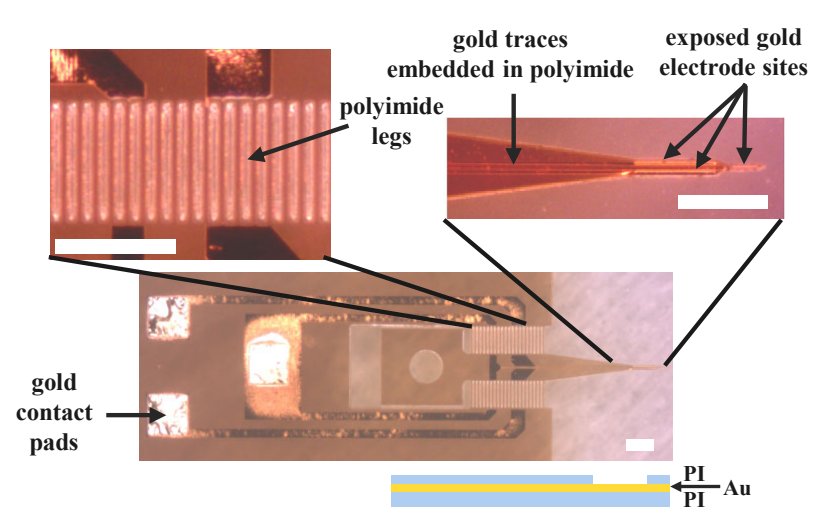

Figure 4: Optical images of the fabricated devices. The device consists of a $10 \mu \mathrm{m}$ polyimidel $200 \mathrm{~nm}$ gold/ $10 \mu \mathrm{m}$ polyimide sandwich structure with the gold exposed at the electrode sites on the tip and at the contact pads. Scale bars $=300 \mu \mathrm{m}$.

\section{RESULTS}

The device was first characterized in benchtop tests in order to answer the following questions before in vivo testing: 1. Does the device tip pierce the brain tissue without buckling? 2. Does the device trigger mechanism work as designed? 3. Are the electrode sites functional?

\section{Benchtop Mechanical and Electrical Characterization}

The first critical component of the device is the probe tip, which is designed to be as flexible as possible while still being able to pierce through brain tissue. Insertion into freshly sacrificed rat pup brains demonstrated that the chosen dimensions could resist buckling (Figure 5). This validated the mechanical model used to design the tip geometry. (a)

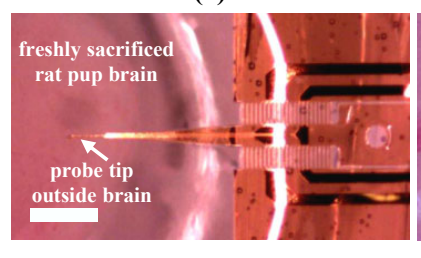

(b)

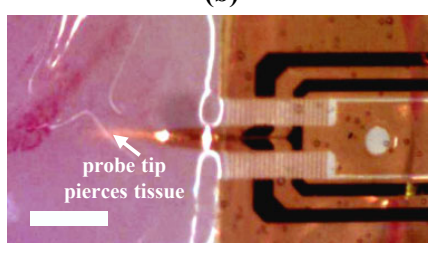

Figure 5: In vitro piercing tests to demonstrate that the probe tips can pierce brain tissue without buckling. (a) The probe tip lies just outside a rat pup brain. (b) As the probe is pushed in, the probe tip pierces the brain tissue without buckling. Scale bars $=1 \mathrm{~mm}$.

The second critical component of the device is the trigger mechanism consisting of the device legs that are deflected to store energy prior to insertion. The legs are then held in place in this stored-energy configuration (Figure 6a) using a water- or thermally-soluble glue, which allows for triggering on-demand. Here we used a polyethylene glycol (PEG) hydrogel that dissolves acutely in saline. PEG was chosen because of its biocompatibility and tunable degradation times [12]. To demonstrate the triggering functionality, we applied saline to the PEG-glued probe, causing the glue to dissolve and the probe body to trigger and advance (Figure 6b). These tests validated the mechanical models used to design the trigger structure geometry. 
(a)
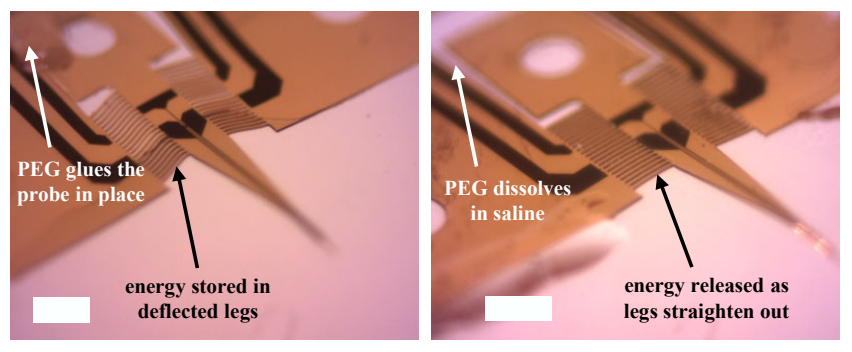

Figure 6: Benchtop triggering test. (a) The pulled-back probe body is held in place with PEG, storing energy in the deflected legs. (b) The PEG is dissolved by adding saline, thereby straightening the legs and pushing the tip forward by approximately $50 \mu \mathrm{m}$. Scale bars $=500 \mu \mathrm{m}$.

The third critical component of the device is the electrical functionality of the electrode sites. We measured the impedance of the electrode sites in saline (using an Agilent 4294A precision impedance analyzer) to be $\sim 70-400 \mathrm{k} \Omega$ at $1 \mathrm{kHz}$, which is within the typical range for recording electrodes.

The benchtop tests above, thus, demonstrate successful mechanical and electrical characterization of the various critical components of the device.

\section{In Vivo Testing - Electrical Functionality}

To demonstrate electrical functionality of the electrodes in an in vivo setting, we inserted untriggered probes in the motor cortex of adult female Long Evans rats. Neural data were recorded intraoperatively under ketamine/xylazine anesthesia upon initial insertion of the device using a commercially available recording system (Tucker Davis Technologies) and single unit waveforms were discriminated using time-amplitude windows. Figure 7 shows a pile plot of the sorted waveform (action potentials) and the distribution of the inter-spike intervals recorded. These preliminary tests demonstrate the efficacy of the device in recording neural activity in an acute in vivo setting.

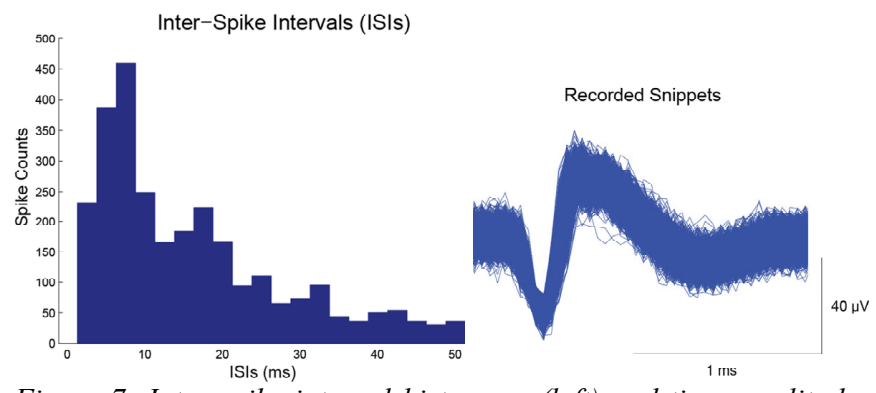

Figure 7: Inter-spike interval histogram (left) and time-amplitude sorted waveforms (right) recorded intra-operatively immediately following device insertion.
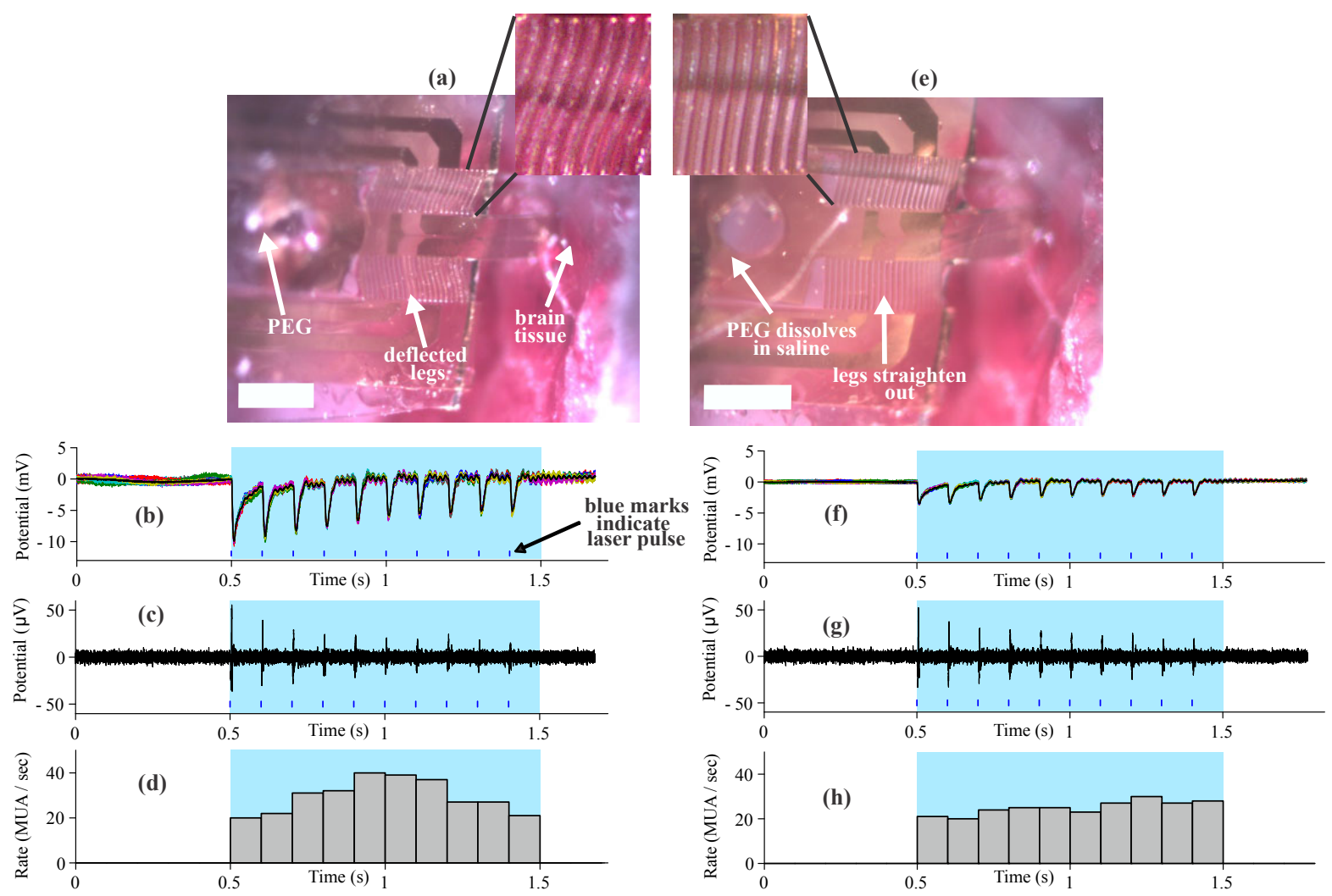

Figure 8: In vivo acute recordings of neural activity in the medial prefrontal cortex of a Thy1-ChR2-YFP optogenetically active mouse. (a) The probe is inserted into the mouse brain in the deflected configuration (Scale bar $=500 \mu m$ ). (b)-(d) Neural activity (Local Field Potentials (LFPs) and Multi-Unit Activity (MUA)) is then recorded closely following 20 laser stimulation trains (laser wavelength = 473 $n m)$. (e) The probe is then triggered causing the tip to pierce $\sim 50 \mu \mathrm{m}$ deeper into the brain. (f)-(h) Neural activity is recorded again in response to the same laser stimulation as in (b)-(d). LFPs ((b),(f)), MUA ((c),(g)) and MUA histograms ((d),(h)) in response to the laser pulses all indicate successful electrical recording both before and after probe deployment. 


\section{In Vivo Testing - Integrated Functionality}

Finally, we confirmed the integrated electrical and mechanical functionality of the probes under acute in vivo conditions in the medial prefrontal cortex of anesthetized (ketamine/xylazine) transgenic Thy1-ChR2-YFP mice. We inserted the probe in the deflected configuration (Figure 8a), and recorded neural activity (Figures 8b-d) closely following 20 laser stimulation trains (each train consisting of 10 pulses at $10 \mathrm{~Hz}$ with $5 \mathrm{~ms}$ pulse duration, and an inter-train interval of $5 \mathrm{~s}$ ) delivered using a stationary optic fiber inserted adjacent to our probe. The probe was then triggered by applying saline to the PEG glue causing the tip to pierce $\sim 50 \mu \mathrm{m}$ deeper into the brain (Figure 8e). Neural activity was recorded again (Figures 8f-h) in response to similar laser stimulation as in Figures b-d. Local field potentials (LFPs, $1-1000 \mathrm{~Hz}$ filter settings, $3 \mathrm{kHz}$ sampling frequency) (Figures 8b,f) indicate successful electrical recordings before and after deployment (colored lines indicate individual recordings from each of the 20 laser trains while the black line indicates the average of these 20 recordings). The LFP amplitude decreases after deployment in Figure $8 \mathrm{f}$ as expected since the probe tip moves deeper into the brain and further away from the stationary source of laser stimulation. Simultaneously with LFPs, we successfully recorded multi-unit activity (MUA) in response to laser pulses both before and after probe deployment (raw traces in Figures 8c,g, filter settings $0.3-8 \mathrm{kHz}, \sim 50 \mathrm{kHz}$ sampling frequency). Histograms for quantification across trials (Figures 8d,h) confirmed the reliability of the evoked activity over a total of the 20 laser stimulation trains. To ensure physiological origin of the recorded activity, we raised the stimulation frequency to $100 \mathrm{~Hz}$ resulting in loss of the correlation between stimulation and neural response as reported previously [13] (data not shown). We also performed a negative control experiment following the animal tests by immersing the probes in $0.9 \%$ sodium chloride solution and detected no response following optical stimulation (data not shown).

These tests demonstrate successful in vivo triggering and electrical recording of neural activity in acute conditions, paving the way for chronic triggering and recording tests using our device.

\section{CONCLUSIONS}

We present the development and successful testing of a new type of reconfigurable neural probe with small dimensions, designed to record neural activity for extended periods of time. Currently, technologies to do this are limited by both acute vascular injury and prolonged tissue reaction, the latter mediated by the body's immune reaction to foreign objects. The combination of these factors limits the reliability of single-unit recording from the brain. Our probe aims to advance beyond the tissue undergoing an immune reaction by changing shape at a time well after initial insertion into the brain, with sufficiently small tip dimensions so as not to trigger further reaction. We have demonstrated successful triggering and electrical recordings from this device in an acute setting in the rodent brain. This technology holds promise for creating chronic interfaces for recording stable neural activity.

\section{ACKNOWLEDGEMENTS}

This work was funded by the NSF Engineering Research Center on Sensorimotor Neural Engineering (NSF-1028725). All devices were fabricated in the Microsystems Technology Laboratories (MTL) at MIT. The authors would like to thank the MTL staff for their support, Dr. Thibault Honegger for providing the rat pup brains, Dr. Jorg Scholvin for polyimide fabrication process advice, Dennis Ward for help with the laser-cut polyimide prototypes, and Abubakar Abid for help with the post-fabrication device processing. Travel support has been generously provided by the Transducer Research Foundation.

\section{REFERENCES}

[1] D.R. Kipke, W. Shain, G. Buzsáki, E. Fetz, J. Henderson, J. Hetke, G. Schalk, "Advanced neurotechnologies for chronic neural interfaces: New horizons and clinical opportunities," Journal of Neuroscience, vol. 28, pp. 11830-11838, Nov 2008.

[2] V. S. Polikov, P. A. Tresco, W. M. Reichert, " Response of brain tissue to chronically implanted neural electrodes," Journal of Neuroscience Methods, vol. 184, pp. 1-18, Oct 2005.

[3] S. Takeuchi, D. Ziegler, Y. Yoshida, K. Mabuchi, T. Suzuki, "Parylene flexible probes integrated with microfluidic channels," Lab on a Chip, vol. 5, pp. 519-523, Apr 2005.

[4] L. Spataro, J. Dilgen, S. Retterer, A. J. Spence, M. Isaacson, J. N. Turner, W. Shain, "Dexamethasone treatment reduces astroglia responses to inserted neuroprosthetic devices in rat neocortex," Experimental Neurology, vol. 194, pp. 289-300, Apr 2005.

[5] P. Stice, J. Muthuswamy, "Assessment of gliosis around moveable implants in the brain," J. Neural Eng., vol. 6, 2009.

[6] J. P. Seymour, D. R. Kipke, "Neural probe design for reduced tissue encapsulation in CNS," Biomaterials, vol. 28, pp. 35943607, 2007.

[7] P. Stice, A. Gilletti, A. Panitch, J. Muthuswamy, "Thin microelectrodes reduce GFAP expression in the implant site in rodent somatosensory cortex," J. Neural. Eng., vol. 4, pp.42-53, 2007.

[8] T. D. Y. Kozai, N. B. Langhals, P. R. Patel, X. Deng, H. Zhang, K. L. Smith, J. Lahann, N. A. Kotov, and D. R. Kipke, "Ultrasmall implantable composite microelectrodes with bioactive surfaces for chronic neural interfaces," Nat Mater, vol. 11, no. 12, pp. 1065-1073, Dec. 2012.

[9] J. Muthuswamy, M. Okandan, A. Gilletti, M. S. Baker, T. Jain, "An array of microactuated microelectrodes for monitoring single-neuronal activity in rodents," IEEE Transactions on Biomedical Eng., vol. 52, no. 8, pp. 1470-77, Aug 2005.

[10] D. Engert, K. Najafi, "New class of chronic recording multichannel neural probes with post-implant self-deployed satellite recording sites," IEEE Transducers, 2011.

[11] D. H. Szarowski, M. D. Andersen, S. Retterer, A. J. Spence, M. Isaacson, H. G. Craighead, J. N. Turner, and W. Shain, "Brain responses to micro-machined silicon devices," Brain Research, vol. 983, no. 1-2, pp. 23-35, Sep. 2003.

[12] N. A. Peppas, J. Z. Hilt, A. Khademhosseini, R. Langer, "Hydrogels in biology and medicine: from molecular priciples to bionanotechnology," Advanced Materials, vol. 18, pp. 1345-1360, 2006.

[13] P. Anikeeva, A. S. Andalman, I. Witten, M. Warden, I. Goshen, L. Grosenick, L. A. Gunaydin, L. M. Frank, and K. Deisseroth, "Optetrode: a multichannel readout for optogenetic control in freely moving mice," Nat Neurosci, vol. 15, no. 1, pp. 163-170, Jan. 2012.

\section{CONTACT}

*Joel Voldman, tel: +1-617-253-2094; voldman@mit.edu 